Check for updates

Cite this: RSC Adv., 2019, 9, 32313

Received 10th September 2019 Accepted 30th September 2019

DOI: $10.1039 / c 9 r a 07256 j$

rsc.li/rsc-advances

\section{Formation of submicron-sized silica patterns on flexible polymer substrates based on vacuum ultraviolet photo-oxidation $\uparrow$}

\author{
Cheng-Tse Wu, (D) a Ahmed I. A. Soliman, (D) ab Toru Utsunomiya, (D) a Takashi Ichii ${ }^{a}$ \\ and Hiroyuki Sugimura*a
}

Formation of precise and high-resolution silica micropatterns on polymer substrates is of importance in surface structuring for flexible device fabrication of optics, microelectronic, and biotechnology. To achieve that, substrates modified with affinity-patterns serve as a strategy for site-selective deposition. In the present paper, vacuum ultraviolet (VUV) treatment is utilized to achieve spatially-controlled surface functionalization on a cyclo-olefin polymer (COP) substrate. An organosilane, 2,4,6,8tetramethylcyclotetrasiloxane (TMCTS), preferentially deposits on the functionalized regions. Welldefined patterns of TMCTS are formed with a minimum feature of $\sim 500 \mathrm{~nm}$. The secondary VUV/(O)treatment converts TMCTS into $\mathrm{SiO}_{x}$, meanwhile etches the bare COP surface, forming patterned $\mathrm{SiO}_{x} /$ COP microstructures with an average height of $\sim 150 \mathrm{~nm}$. The resulting $\mathrm{SiO}_{x}$ patterns retain a good copy of TMCTS patterns, which are also consistent with the patterns of photomask used in polymer affinitypatterning. The high quality $\mathrm{SiO}_{x}$ patterns are of interests in microdevice fabrication, and the hydrophilicity contrast and adjustable heights reveal their potential application as a "stamp" for microcontact printing $(\mu \mathrm{CP})$ techniques.

\section{Introduction}

Flexible functional devices based on polymer substrates have attracted much attention for their great potential applications. ${ }^{1-3}$ Various oxide films have been formed on polymer substrates to achieve their functionality. ${ }^{4-7}$ In particular, patterning of oxide thin films is of great significance in the development of sensor arrays, ${ }^{8}$ memory arrays, ${ }^{9}$ integrated electronics, ${ }^{10}$ solar cell, ${ }^{11}$ and so forth. In general, the formation for such oxide thin films should be conducted in mild conditions to remain the optical and mechanical properties of polymer substrate, therefore, the traditional micropatterning strategies involving erosive chemicals or high temperature conditions are no longer favoured., ${ }^{72,13}$

Silica micron/submicron-sized patterns were reported to be fabricated on substrates for the application in patterning techniques, ${ }^{\mathbf{1 3}, \mathbf{1 4}}$ optics, ${ }^{\mathbf{1 5 , 1 6}}$ optoelectronics, ${ }^{17}$ microelectronics, ${ }^{18}$ sensors, ${ }^{19}$ and biotechnology. ${ }^{20,21}$ A top-down approach for the silica patterning applicable to arbitrary substrates was reported, which sputtered silica films onto photoresist-patterned

${ }^{a}$ Department of Materials Science and Engineering, Graduate School of Engineering, Kyoto University, Kyoto 606-8501, Japan. E-mail: sugimura.hiroyuki.7m@kyoto-u.ac. jp; Tel: +81-75-753-9131

${ }^{b}$ Chemistry Department, Faculty of Science, Assiut University, Assiut 71516, Egypt

† Electronic supplementary information (ESI) available: Additional optical images of photomask, FTIR spectra. See DOI: 10.1039/c9ra07256j surface. ${ }^{21}$ Other approaches involving bottom-up techniques were reported, in which the use of photoresist was not required. ${ }^{\text {20,22,23 }}$ A poly(dimethylsiloxane) (PDMS) with desired shapes was attached onto the surface to build a "microchannel", subsequently a silica precursor was then injected into the PDMS channel and selectively deposited on the substrates. ${ }^{20}$ Although this site-selective silica treatment was applicable to various substrates, non-continuous patterns such as dotted patterns could not be achieved by this mean.

Site-selective oxide deposition was reported as a useful technique for patterning oxides on substrates, and the achievable shapes and resolution were related to the photomask. Patterns of $\mathrm{SiO}_{x}$ (ref. 22) and $\mathrm{TiO}_{2}$ (ref. 24) were successfully prepared on silicon substrates via the assistance of selfassembled monolayers (SAM). SAM-covered silicon substrates were patterned by UV irradiation with a photomask, causing an affinity contrast between the UV-treated and non-treated regions. The oxide deposits could thus selectively grow guided by the affinity-patterns. In contrast, polymer substrates are commonly known as non-polar or poorly polar materials which have quite "inert" surface for the deposition of oxide layers. To achieve an affinity-pattern for site-selective deposition, several photochemical treatments could be used to tailor the surface of polymer substrates. ${ }^{25-27} \mathrm{~A}$ famous example was given, which applied a UV-activated oxidation to arbitrary polymer substrates in an ammonium persulfate (APS) solution. ${ }^{27}$ The sulfate anion groups $\left(-\mathrm{OSO}_{3}{ }^{-}\right)$were implanted on the outermost surface of 
the polymer substrates, resulting in the increase of hydrophilicity. The spatial-controlled hydrophilic treatment was successfully conducted by UV irradiation through a photomask. The affinity-patterned polymer surface was used for the selective deposition of $\mathrm{SiO}_{x}{ }^{13} \mathrm{TiO}_{2}$ (ref. 28) and $\mathrm{ZnO}^{29}$ However, the affinity-patterning from this technique was conducted in liquid phase, in which the excitation UV light might be refracted to cause inconsistency, and photoexcited APS molecules in the bulk liquid might lead to a broadening of oxidized area, which constrained the achievable resolution. Moreover, the photoactive chemicals were required for such surface-tailoring techniques, which caused complexity in operation, and also generated chemical wastes. Therefore, a dry method, which does not require oxidative chemical reagents, is considered to be an alternative oxidation methodology.

VUV ( $\lambda=172 \mathrm{~nm}, \mathrm{Xe}_{2}$ excimer lamp) light is strongly absorbed by $\mathrm{O}_{2}$, generating active species such as ozone and atomic oxygen. ${ }^{30-33}$ VUV light and the active oxygen species (VUV/(O)) are highly oxidative to organics. ${ }^{7}$ Such VUV/(O)treatment is widely used in photo-oxidation including the removal of organics and the surface hydrophilic treatment. ${ }^{25,30,31}$ Polar functional groups ( $\mathrm{CHO}, \mathrm{CH}_{2} \mathrm{OH}, \mathrm{COOH}$, etc.) could thus be decorated onto a cyclo-olefin polymer (COP) substrate. ${ }^{25}$ These Polar functional groups favoured the oxide depositions since they could participate in the condensation polymerization of sol-gel depositions. ${ }^{34,35}$ Moreover, the VUV/(O)-generated functional groups were reported to be good reaction sites for the silanization. ${ }^{36-38}$ Thin films of an organosilane - 2,4,6,8tetramethylcyclotetrasiloxane (TMCTS) were fabricated on the $\mathrm{VUV} /(\mathrm{O})$-treated polymer substrates. ${ }^{36,37}$ The TMCTS thin films were further converted into $\mathrm{SiO}_{x}$ by the secondary $\mathrm{VUV} /(\mathrm{O})$ irradiation..$^{36-38}$ The resulting $\mathrm{SiO}_{x}$ layers were flat and strongly attached on the polymer surface due to the chemical bonding. Inspired by the above studies, we expect a precise and highresolution TMCTS patterning on the $\mathrm{VUV} /(\mathrm{O})$-patterned COP substrate. The secondary $\mathrm{VUV} /(\mathrm{O})$-irradiation converts the TMCTS patterns to $\mathrm{SiO}_{x}$ patterns.

In this study, we demonstrated submicron-sized $\mathrm{SiO}_{x}$ patterns on the COP substrates by VUV/(O)-treatments (Fig. 1). In the first part, we introduced a $\mathrm{VUV} /(\mathrm{O})$-treatment for spatially-controlled surface modification on COP surface. In the second part, adsorption of TMCTS on the pristine or VUV/(O)modified COP surface was investigated by Fourier transform infrared spectroscopy (FTIR). Photo-oxidation converting TMCTS into $\mathrm{SiO}_{x}$ was studied by using FTIR and X-ray photoelectron spectroscopy (XPS). In the last part, site-selective deposition of TMCTS was conducted. Formation of TMCTS patterns on the affinity-patterned COP substrates was observed by scanning electron microscope (SEM). After the secondary $\mathrm{VUV} /(\mathrm{O})$-treatment, the TMCTS patterns were converted into silica patterns.

\section{Experimental}

\section{$\mathrm{VUV} /(\mathrm{O})$-patterning on COP}

$\mathrm{VUV} /(\mathrm{O})$-treatment was conducted using VUV light $(\lambda=172 \mathrm{~nm})$ from a $\mathrm{Xe}_{2}$ excimer lamp (UER 20-172 V, Ushio Inc.). The power (a)

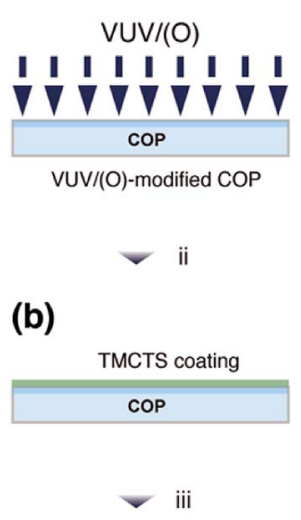

(c)

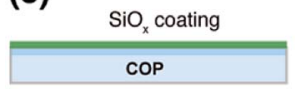

(d)

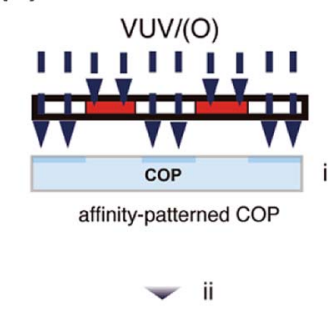

(e)

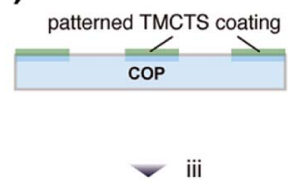

(f)

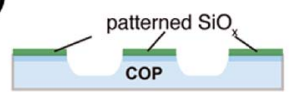

Fig. 1 Schematic illustration of (a) VUV/(O)-modification on COP surface, (b) TMCTS deposition on the modified COP surface, (c) $\mathrm{SiO}_{x}$ thin films formed by VUV/(O)-photo-oxidation, (d) affinity-patterning by $\mathrm{VUV} /(\mathrm{O})$-treatment through a photomask, (e) site-selective deposition of TMCTS on the affinity-patterned COP surface, (f) $\mathrm{SiO}_{x}$ microstructures on the COP substrate.

of VUV light was measured as $10 \mathrm{~mW} \mathrm{~cm}^{-2}$ with $\mathrm{N}_{2}$ purging at a distance of $5 \mathrm{~mm}$ from the window of VUV lamp. All the VUV/ (O)-treatments were carried out at this distance. A cyclo-olefin polymer (COP, ZF16-188, ZeonorFilm®, ZEON Corporation) sheet with a thickness of $0.188 \mathrm{~mm}$ was cut into the size of $2 \mathrm{~cm}$ $\times 2 \mathrm{~cm}$ for the use as a substrate. The protective films on the COP substrates were peeled off, then the newly exposed COP surface was VUV/(O)-treated under $10^{3}$ Pa dried air environment for $10 \mathrm{~min}$. The $\mathrm{VUV} /(\mathrm{O})$-generated functional groups were characterized by attenuated total reflectance FTIR (ATR-FTIR).

The affinity-patterning on the COP substrate was performed by $\mathrm{VUV} /(\mathrm{O})$-treatment through a photomask. The photomask consists of a $100 \mathrm{~nm}$-thick chromium coating with patterns on a $2 \mathrm{~mm}$-thick quartz plate (93\% transparency for light at 172 $\mathrm{nm})$. After affinity-patterning, the changes in topography and phase were measured by AFM with AC-mode.

\section{TMCTS-treatment and silica thin film formation}

The procedure of TMCTS-treatment was described as below. A precursor solution was prepared by mixing 2,4,6,8-tetramethylcyclotetrasiloxane (TMCTS, 99\%, Alfa-Aesar), ethanol (99.5\%, Nacalai Tesque), and ultrapure water (resistivity of 18.2 $\mathrm{M} \Omega \mathrm{cm}$ ) in the amount of $5 \mathrm{~mL}, 10 \mathrm{~mL}, 0.1 \mathrm{~mL}$, respectively. The COP substrates were located with the precursor solution in a $120 \mathrm{~cm}^{3}$ perfluoroalkoxy alkane jar (PFA jar, ARAM Corporation) and heated in an oven maintaining the temperature at $80{ }^{\circ} \mathrm{C}$ for 24 hours. The physically adsorbed precursor molecules were washed away through the ultrasonic cleaning in ethanol for 1 hour. The resulting TMCTS thin films were further treated by $\mathrm{VUV} /(\mathrm{O})$ under $10^{3} \mathrm{~Pa}$ dried air environment for a series of 
irradiation time. The characterization of TMCTS thin films before and after $\mathrm{VUV} /(\mathrm{O})$-treatment was conducted by WCA measurement, XPS and ATR-FTIR.

\section{Site-selective TMCTS deposition on affinity-patterns}

TMCTS-treatment was applied on the affinity-patterned COP substrates, which were prepared as previously described. After the TMCTS-treatment, the substrates were ultrasonically cleaned in ethanol for 1 hour to leave a TMCTS pattern. Scanning electron microscope (SEM) equipped with an energy dispersive X-ray spectroscopy (EDX) was used to study the siteselective adsorption of TMCTS. Afterwards, the TMCTS patterns were further treated by $\mathrm{VUV} /(\mathrm{O})$ to form $\mathrm{SiO}_{x}$ micro arrays.

\section{Analytical techniques}

XPS measurement was performed using a photoelectron spectrometer (ESCA-3400 system, Kratos Analytical) equipped with $\mathrm{Mg} \mathrm{K} \mathrm{K}_{\alpha} \mathrm{X}$-ray source (emission current $10 \mathrm{~mA}$, accelerate voltage $10 \mathrm{kV}$ ) to investigate the chemical changes of COP surface during the VUV/(O)-treatment and TMCTS adsorption. High resolution spectra were observed at $\mathrm{C} 1 \mathrm{~s}$ and $\mathrm{Si} 2 \mathrm{p}$ regions with each energy step of $0.1 \mathrm{eV}$. The obtained XPS spectra were referenced to $\mathrm{C}-\mathrm{C}(285.0 \mathrm{eV})$ of $\mathrm{C}$ 1s spectra. FTIR (Excalibur FTS-3000, Digilab) was used to identify the formation of VUV/ (O)-generated functional groups and the adsorption of organosilane, which could support the results of XPS. The IR measurement was conducted in ATR-mode using a germanium ATR crystal (GATR, Harrick Scientific Product) with a reflection angle of $65^{\circ}$. The IR spectra were received in the range of 2300$900 \mathrm{~cm}^{-1}$ with a resolution of $2 \mathrm{~cm}^{-1}$. WCA values were obtained with a static contact angle meter (DM 500, Kyowa Interface Science Co. Ltd.), where the volume of water droplet was fixed at $1.8 \mu \mathrm{L}$. SEM (S-3500H, Hitachi) and EDX (Delta Plus 3, Kevex/Fisons Instruments Inc.) were used for observing the TMCTS patterns and specifying the existence of silicon element. The SEM images and EDX area analysis were obtained with an electron beam acceleration voltage of $5 \mathrm{kV}$. AFM (MFP-3D, Oxford Instruments) was conducted to visualize the morphology of the TMCTS and silica micropatterns. The images of topography and phase were acquired by AC-mode using aluminium backside coated Si probes (SI-DF-40, Hitachi HiTech Co. Ltd.).

\section{Results and discussion}

\section{VUV/(O)-modification on COP surface}

ATR-FTIR measurement were conducted to verify the surface modification on the COP substrates, as shown in Fig. 2. A band in 1500-1400 $\mathrm{cm}^{-1}$ with two split peaks at $1464 \mathrm{~cm}^{-1}$ and $1447 \mathrm{~cm}^{-1}$ could be attributed to $\mathrm{CH}_{2}$ deformation vibrations. ${ }^{25,26} \mathrm{VUV} /(\mathrm{O})$-treatment introduced absorption bands in the frequency range of $1800-1600 \mathrm{~cm}^{-1}$, which was attributed to carbonyl groups $(\mathrm{C}=\mathrm{O}) .{ }^{25,26}$ An appearance of absorption bands at $1800-1500 \mathrm{~cm}^{-1}$ contained doublet structure, where the sharper peak was centred at $1711 \mathrm{~cm}^{-1}$ and the broad band was

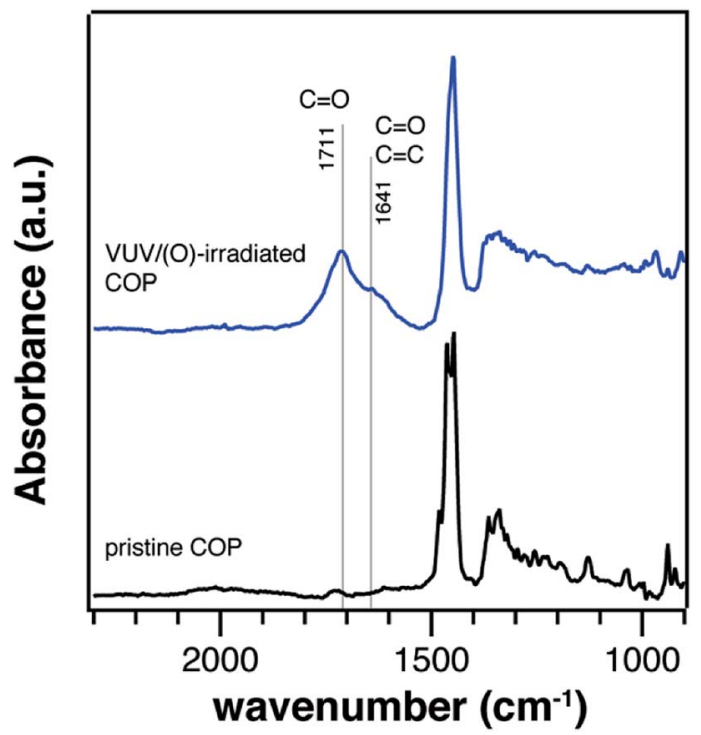

Fig. 2 ATR-FTIR spectra of COP before and after VUV/(O)-irradiation for $10 \mathrm{~min}$.

centred at $1641 \mathrm{~cm}^{-1}$. The sub-peak centred at $1711 \mathrm{~cm}^{-1}$ could be attributed to $\mathrm{C}=\mathrm{O}$ vibrations from aliphatic ketones, aldehydes, and carboxylic acid species, which were resulted from the oxidation of alkyl groups. ${ }^{25,31}$ A broad absorption band at $1641 \mathrm{~cm}^{-1}$ could be attributed to $\mathrm{C}=\mathrm{O}$ from unsaturated ketones and aldehydes, as well as $\mathrm{C}=\mathrm{C}$ of alkene structure. ${ }^{25}$ These unsaturated structures could be attributed to the ring opening reaction and dehydrogeneration. ${ }^{25,26}$ Based on these results, the generation of functional groups on COP surface was confirmed. These polar functional groups are thought to be of importance for the deposition of oxides and silanes.

In this section, we demonstrated that the photochemical modification by $\mathrm{VUV} /(\mathrm{O})$-treatment is effective in obtaining hydrophilicity at the surface. Since the surface modification is induced by $\mathrm{VUV} /(\mathrm{O})$-treatment, patterned surface modification could be achieved through a photomask. By this mean, we expect that the confined surface tailoring and further siteselective nano-architecture could be realized.

Affinity-patterning was performed on COP substrates by applying VUV/(O)-treatment through a photomask with circular patterns. The VUV photons penetrated through the slits and irradiated the COP surface, simultaneously the active oxygen species were generated above the irradiated regions. The dispersion of the active oxygen species was reported to affect the masked regions as well, leading to a slight broadening of oxidized area. ${ }^{39}$ Here, the changes of topography and phase during affinity-patterning were observed by AFM, as shown in Fig. 3. In the first $1 \mathrm{~min} \mathrm{VUV} /(\mathrm{O})$-irradiation through a photomask, the differences between $\mathrm{VUV} /(\mathrm{O})$-irradiated regions and masked regions were clearly identified by the topographic image (Fig. 3a) and phase image (Fig. 3b). The phase image portrays the mapping of mechanical properties on the surface. The phase image (Fig. 3b) shows a distinct difference in mechanical properties between the $\mathrm{VUV} /(\mathrm{O})$-irradiated and the 
(a)

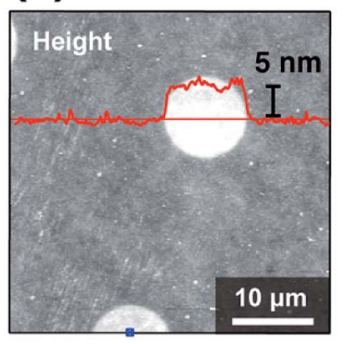

(b)

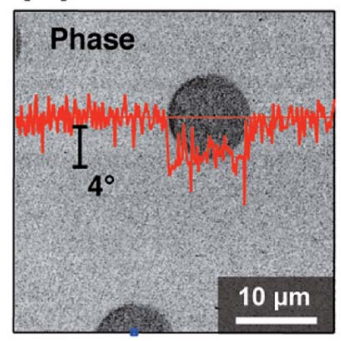

patterned $\mathrm{VUV} /(\mathrm{O})$ for $1 \mathrm{~min}$

(c)

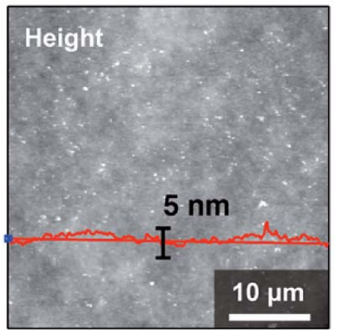

(d)

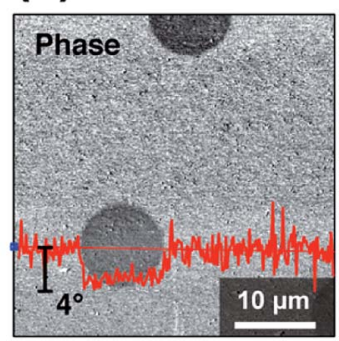

patterned VUV/(O) for $10 \mathrm{~min}$

Fig. 3 AFM topographic images of VUV/(O) affinity-patterning on COP surface for (a) $1 \mathrm{~min}$ and (c) 10 min, respectively. (b) and (d) are corresponding phase images.

masked regions. In the topographic image (Fig. 3a), the VUV/ (O)-irradiated regions were found to be higher than the masked regions, most likely due to the permeation of oxygen into COP and the generation of oxygenated carbon groups. On the other hand, $\mathrm{VUV} /(\mathrm{O})$ is known to etch the irradiated region via photo-degradation of $\mathrm{COP}^{26}$ After $10 \mathrm{~min} \mathrm{VUV} /(\mathrm{O})$ irradiation through a photomask, the differences in surface properties can be still observed in phase image (Fig. 3d), and the COP surface turned into topographically flat (Fig. 3c). Thus, the affinity-patterned COP substrate with little topographic difference was obtained.

\section{TMCTS thin films and photo-oxidation}

The polar functional groups on the COP surface were reported to play a role in the organosilane adsorption since they provide chemical anchoring sites for silanization. ${ }^{36,37,40}$ In Fig. 4, the ATR-FTIR spectra portray the evolution of IR absorption bands after TMCTS-treatments on the pristine COP or $\mathrm{VUV} /(\mathrm{O})$ modified COP substrates. No obvious change was found before (Fig. 4A) and after (Fig. 4B) the TMCTS-treatment applied on the pristine COP substrate. In comparison, several newly generated peaks were found after TMCTS-treatments applied on the VUV/(O)-modified COP substrate, which were shown in Fig. $4 \mathrm{C}$ and D. The COP related IR bands were also detected since the sampling depth can reach to $0.37-0.47 \mu \mathrm{m}$ in region of 1800-1400 $\mathrm{cm}^{-1} \cdot{ }^{41}$ Absorption bands at $2168 \mathrm{~cm}^{-1}$ and $1270 \mathrm{~cm}^{-1}$ corresponded to $\mathrm{Si}-\mathrm{H}$ stretching vibration and $\mathrm{Si}-$ $\mathrm{CH}_{3}$ deformation, respectively. ${ }^{42-44} \mathrm{~A}$ broad absorption band at

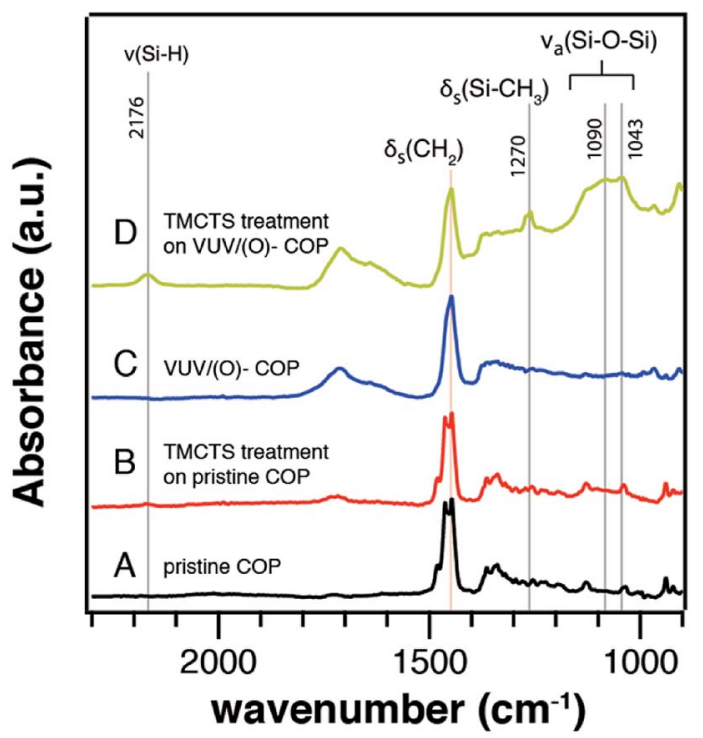

Fig. 4 ATR-FTIR spectra of modified COP surfaces. (A) Pristine COP, (B) pristine COP after TMCTS-treatment, (C) 10 min VUV/(O)-modified COP, and (D) 10 min VUV/(O)-modified COP after TMCTS-treatment.

1160-975 $\mathrm{cm}^{-1}$ was due to $\mathrm{Si}-\mathrm{O}-\mathrm{Si}$ asymmetric stretching vibrations. Compared with the IR spectra of TMCTS monomer (Fig. S2 $\dagger$ ), the obtained TMCTS coatings revealed a broader frequency range of $\mathrm{Si}-\mathrm{O}-\mathrm{Si}$ vibration. This broad frequency range could consist of vibrational modes from intact TMCTS rings at $1113 \mathrm{~cm}^{-1}$ and $1090 \mathrm{~cm}^{-1}$, and silica-like structure with different $\mathrm{Si}-\mathrm{O}-\mathrm{Si}$ bond angles..$^{4-47}$

Based on the IR results, two phenomenon were clarified: (1) the $\mathrm{VUV} /(\mathrm{O})$-modification on the COP surface was crucially important for the further silanization. (2) The broader frequency range of $\mathrm{Si}-\mathrm{O}-\mathrm{Si}$ asymmetric stretching vibration suggested that the intermolecular linkages incorporated in the TMCTS layers on the COP surface, forming the primary Si-O-Si network with siloxane structures.

TMCTS thin films were known to be converted into inorganic $\mathrm{SiO}_{x}$ thin films after VUV/(O)-treatments, where VUV/(O) caused the removal of the methyl terminal groups and formed the network of Si-O-Si bond chains. ${ }^{36,37}$ Our chemically adsorbed TMCTS thin films on the COP surface were treated by VUV/(O) for variant irradiation time. XPS and ATR-FTIR measurements were conducted to elucidate the changes of $\mathrm{VUV} /(\mathrm{O})$-treated TMCTS films, as shown in Fig. 5. The XPS C 1s and Si 2p spectra were shown in Fig. 5a. The intensity of $\mathrm{C}$ 1s decreased with the irradiation time. After $50 \mathrm{~min} \mathrm{VUV} /(\mathrm{O})$-treatment, the atomic ratio of carbon decreased from 0.60 to 0.12 . This remnant of carbon is commonly attributed to contaminant adsorption from the atmospheric environment. ${ }^{48,49}$ In $\mathrm{Si} 2 \mathrm{p}$ spectra, the peak position was gradually shifted from $103.0 \mathrm{eV}$ to $103.9 \mathrm{eV}$. This chemical shift is usually attributed to the chemical conversion from organosilane to amorphous silica. ${ }^{36,42}$ The atomic concentration was also calculated based on the $\mathrm{Si} 2 \mathrm{p}$ and $\mathrm{O} 1 \mathrm{~s}$ spectra. The atomic ratio of $\mathrm{Si}$ to $\mathrm{O}$ was calculated to be $1: 2.7$, which is in good agreement with previously reported $\mathrm{SiO}_{x}$ depositions. ${ }^{13,36}$ ATR-FTIR measurements were also 
(a)
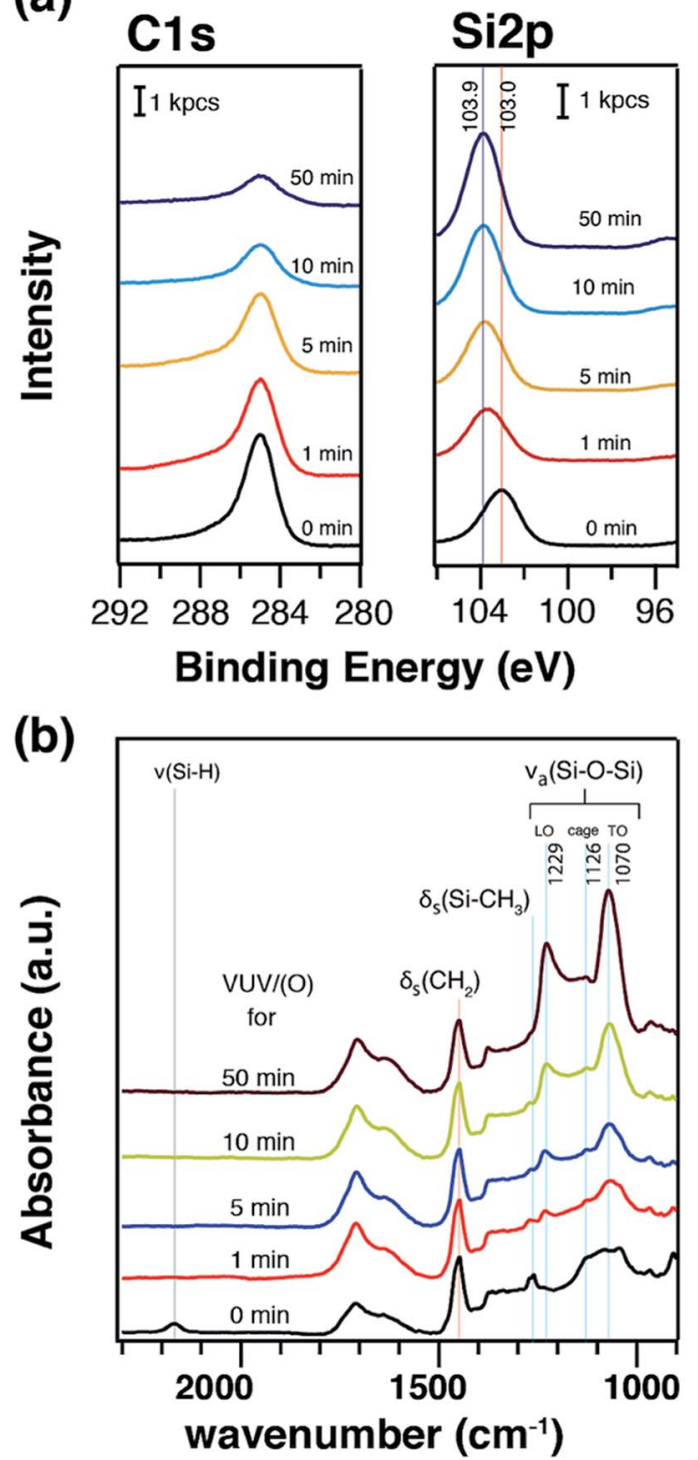

Fig. 5 (a) XPS C1s and Si $2 p$ spectra of TMCTS thin films before and after $\mathrm{VUV} /(\mathrm{O})$-treatment for 1, 5, 10, $50 \mathrm{~min}$. (b) ATR-FTIR spectra of TMCTS before and after VUV/(O)-treatment for 1, 5, 10, 50 min (from bottom to top).

performed to understand the evolution of Si-related bonds, as shown in Fig. 5b. After the first 1 min irradiation, the IR band due to stretching vibration of $\mathrm{Si}-\mathrm{H}\left(\sim 2176 \mathrm{~cm}^{-1}\right)$ vanished. A reduction of band related to $\mathrm{Si}-\mathrm{CH}_{3}$ was also observed. $\mathrm{VUV} /(\mathrm{O})$ treatment was further conducted in longer irradiation time. The peak of $\mathrm{Si}-\mathrm{CH}_{3}$ was largely reduced. The $\mathrm{Si}-\mathrm{O}-\mathrm{Si}$ asymmetric stretching bands, which are in the range of $1160-975 \mathrm{~cm}^{-1}$, were gradually sharpen to $1070 \mathrm{~cm}^{-1}$. This vibration at $1070 \mathrm{~cm}^{-1}$ and another newly generated vibration at $1229 \mathrm{~cm}^{-1}$ are commonly attributed to $\mathrm{Si}-\mathrm{O}-\mathrm{Si}$ vibrations in silica, which were denoted as transverse optical mode (TO) ${ }^{43,50}$ and longitudinal optical vibration mode (LO), ${ }^{50-52}$ respectively. A sub-peak at $1126 \mathrm{~cm}^{-1}$ was also observed. This peak can be attributed to $\mathrm{Si}-\mathrm{O}-\mathrm{Si}$ of caged structures, which are similar to the structures in silsesquioxane polymers. ${ }^{45,47}$ The results from XPS and ATR-FTIR indicate that the methyl groups in TMCTS layers were clearly removed, and the -Si-O-Si- chains were converted into fully relaxed silica network.

Until here, we successfully formed inorganic thin films $\mathrm{SiO}_{x}$ on the COP surface. The static water contact angles (WCA) were also measured for evaluating the surface change. WCA values of (a) pristine COP surface, (b) VUV/(O)-modified COP, adsorbed TMCTS thin films (c) before and (d) after VUV/(O)treatment were shown in Fig. 6. After $10 \mathrm{~min} \mathrm{VUV/(O)-}$ treatment on pristine COP, the WCA values decreased from $96.7^{\circ}$ to $24.3^{\circ}$ (Fig. 6a and b). This modified surface provided sufficient chemical affinity for silanization of TMCTS molecules, which resulted in a good coverage of organosilane thin films. The WCA value of resulting TMCTS thin films was measured to be $95.9^{\circ}$ (Fig. 6c), which is consistent with other reported TMCTS depositions from CVD techniques. ${ }^{36,37,53}$ The increased hydrophobicity is due to the terminal $\mathrm{CH}_{3}$ groups toward the outer surface. ${ }^{37,42,53}$ By the secondary VUV/(O)treatment, the organic moieties were eliminated, resulting in a hydrophilic inorganic surface (Fig. 6d).

\section{Site-selective silanization on affinity-patterns}

The pristine COP surface was found to be chemically inert for the silanization. On the contrary, TMCTS thin films were successfully formed on the VUV/(O)-modified COP substrate due to the polar functional groups. Based on these, we expected that TMCTS molecules could selectively deposit at VUV/(O)modified regions on COP surface. TMCTS-treatment was carried out on the COP substrates with affinity-patterns. The selective depositions were monitored by SEM-EDX (Fig. 7) and AFM (Fig. 8) analysis.

In Fig. 7a, the SEM image illustrates a micro array in circular shapes, as indicated by the white arrow. This contrast is probably due to the difference in height from the selective deposition of TMCTS molecules. The height measurement was also made by AFM, which will be described later. EDX analysis was conducted to elucidate the elements in the circular patterns (Area 1) and out of the circular patterns (Area 2). In Fig. 7b, the upper panel illustrates the EDX spectra in Area 1, which represent the corresponding position in the SEM image. A peak at $\sim 1.74 \mathrm{keV}$ was observed. This position is attributed to Si Ka $\mathrm{X}$-ray line. In contrast, the lower panel illustrating the EDX spectra of Area 2 revealed only noises near the position of Si K $\alpha$ X-ray line. It is suggested that TMCTS with the Si element deposited selectively in the circular-shaped regions, which matched the hydrophilic regions of the affinity-patterned COP surface. In Fig. 7c and d, high-resolution TMCTS line patterns were also performed on the COP surface with line-shaped hydrophilic patterns.

The TMCTS-micropatterned substrates were further treated by $\mathrm{VUV} /(\mathrm{O})$ to convert TMCTS to $\mathrm{SiO}_{x}$. The morphology and height histogram of TMCTS micropatterns before and after $\mathrm{VUV} /(\mathrm{O})$-treatment are shown in Fig. 8. The height of the TMCTS micropatterns is $\sim 20 \mathrm{~nm}$ by the height histogram. After VUV/(O)-treatment, TMCTS patterns were converted to 
(a)

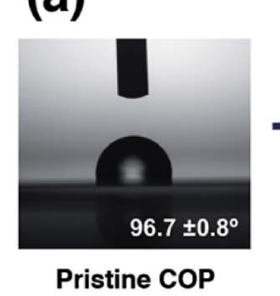

(b)

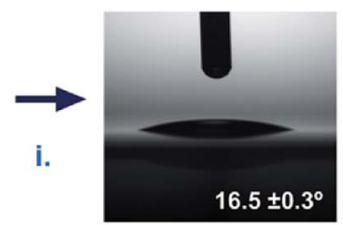

VUV(O)-treated COP (c)

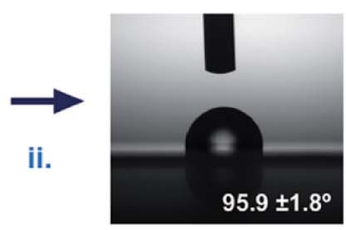

TMCTS films (d)

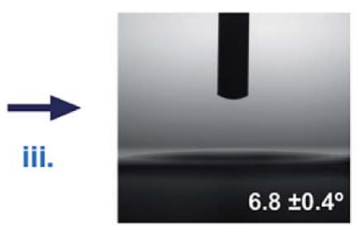

VUV(O)-treated TMCTS films

Fig. 6 WCA images and values of (a) pristine COP, (b) VUV/(O)-modified COP, (c) TMCTS thin films and (d) VUV/(O)-treated TMCTS thin films.

$\mathrm{SiO}_{x}$ patterns. The thickness of TMCTS films is thought to be reduced after $\mathrm{VUV} /(\mathrm{O})$-treatment due to the removal of organic parts and the condensation of silica structures. ${ }^{22,36,37,43}$ However, the bare COP surface was etched since VUV/(O) caused the degradation of $\mathrm{COP}$, as previously reported..$^{25,26}$ On the other hand, the regions covered by TMCTS (later converted to $\mathrm{SiO}_{x}$ ) were protected from the photo-degradation since the active oxygen species cannot reach the masked COP. Although a few amounts of the VUV photons could penetrate the $\mathrm{SiO}_{x}$ layers and might dissociate the $\mathrm{C}-\mathrm{O}-\mathrm{Si}$ bonds, the exfoliation of silica thin films from COP was not found in the previously demonstrated XPS and FTIR results, which is also in good agreement with previously reported silica thin films. ${ }^{36,37}$ Therefore, the silica micro arrays with COP pillar-like supports were fabricated. The average height of the microstructures of $\mathrm{SiO}_{x} / \mathrm{COP}$ pillars is $\sim 150 \mathrm{~nm}$ obtained from the height histogram. Since the TMCTS adsorptions were strictly confined in the affinity-patterned regions, and $\mathrm{SiO}_{x}$ masked COP was not changed by the further $\mathrm{VUV} /(\mathrm{O})$ treatment, the shapes of the micropatterns remained consistent with affinity-patterned regions even after the secondary $\mathrm{VUV} /(\mathrm{O})$-treatment.

The optical micrographs show that high quality $\mathrm{SiO}_{x} / \mathrm{COP}$ micro arrays on the flexible substrates were successfully fabricated over a large area, as shown in Fig. 9. The $\mathrm{SiO}_{x}$ micropatterns are in good agreement with the slit size of the photomask used in the affinity-patterning. High-resolution $\mathrm{SiO}_{x}$ micro arrays fabricated on flexible substrates are of (a)

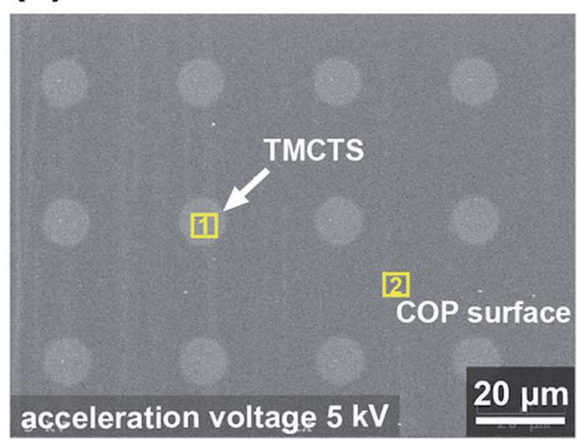

(c)

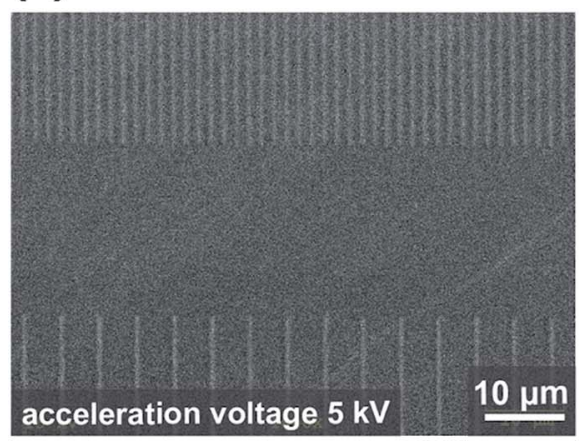

(b)

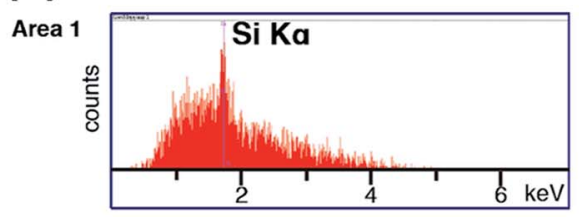

Area 2

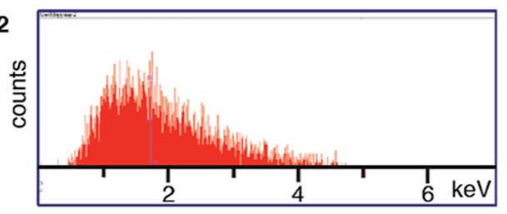

(d)

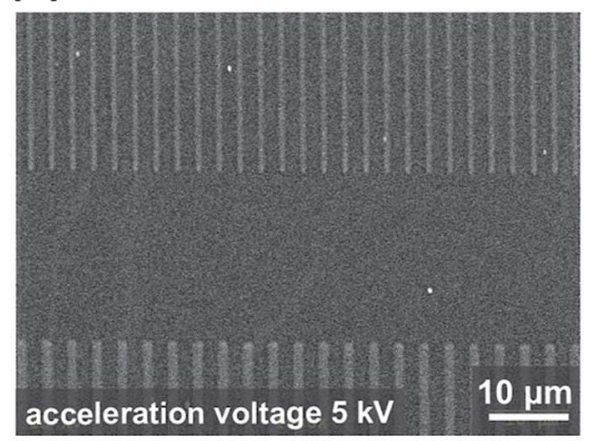

Fig. 7 SEM-EDX analysis of TMCTS patterns on COP surface. (a) SEM image illustrates the patterned regions, marked as Area 1 and Area 2. (b) Area EDX analysis of corresponding position in the SEM image. Line patterns of various features in (c) domains, which have (interval, line width) of $(1 \mu \mathrm{m}, 0.5 \mu \mathrm{m})$ and $(4 \mu \mathrm{m}, 0.5 \mu \mathrm{m})$. (d) Domains, which have (interval, line width) of $(2 \mu \mathrm{m}, 0.5 \mu \mathrm{m})$ and $(2 \mu \mathrm{m}, 1 \mu \mathrm{m})$. 
(a)

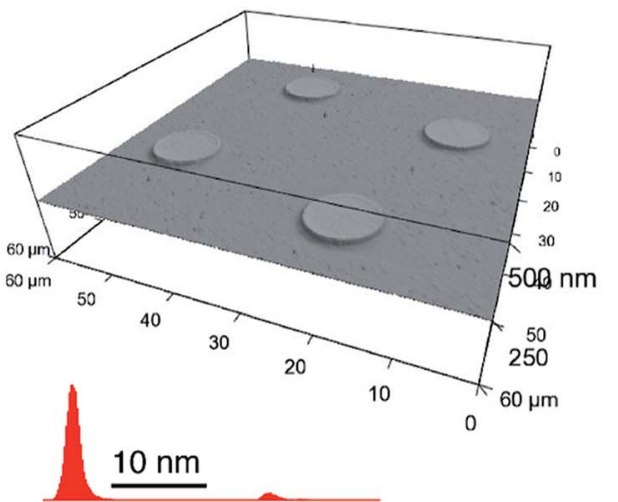

(c)

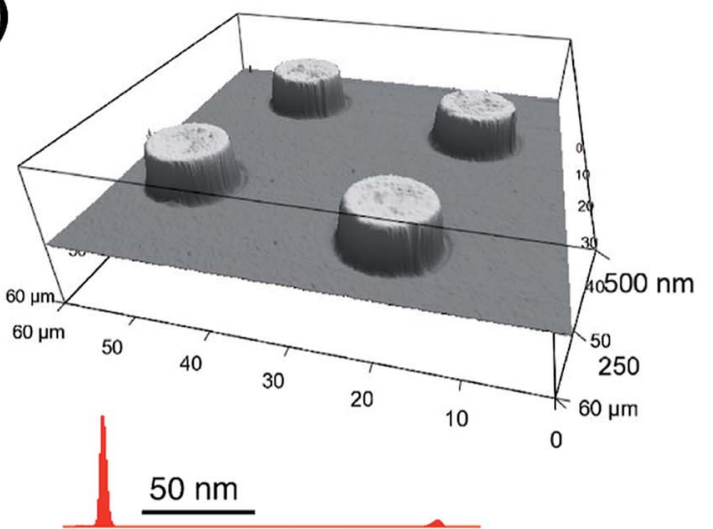

(b)

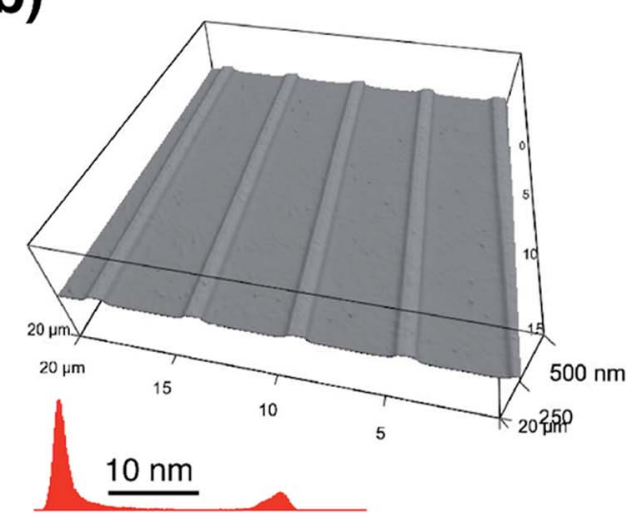

(d)

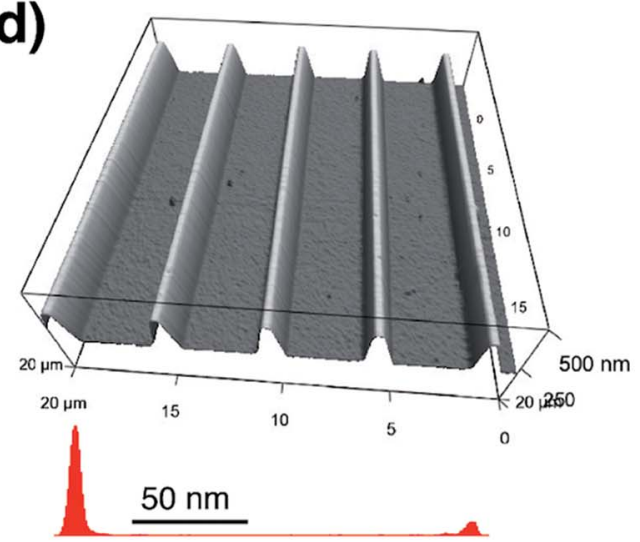

Fig. 8 AFM 3D-topographic images and the corresponding height histogram of TMCTS patterns ( $a$ and $b$ ) before and (c and d) after VUV/(O)treatment for $50 \mathrm{~min}$, where $(a$ and $c)$ represent the circular pattern, and $(b$ and $d$ ) represent the line pattern.

great importance for the design and creation of flexible microdevices in optics, electronics, optoelectronics and biotechnology, which were mentioned in the introduction. Since the resolution is achievable in sub- $\mu \mathrm{m}$ scale, the resulting patterns have great potential in future formation of metamaterials. ${ }^{54}$ Furthermore, our silica-patterned polymer substrates might be devoted to further patterning techniques. There is a distinct height difference between $\mathrm{SiO}_{x}$ and the COP substrate. This enables us to transfer ink films onto $\mathrm{SiO}_{x}$ patterns without contaminating the COP substrate. By dipping the silica-patterned polymer substrates to the (3-aminopropyl) triethoxysilane (APTES) gel films, APTES is immobilized on silica patterns with Si-O-Si linkage to form APTES patterns, which are useful in patterning of proteins. ${ }^{13}$ Similar process can be conducted by transferring metal oxide precursor to silica patterns for the direct formation of metal oxide patterns, which should receive great interest of electronic device fabrication on flexible polymer substrates. ${ }^{29}$ Another potential application is to use the $\mathrm{SiO}_{x}$ micropatterned COP as a "stamp" for the micro contact printing $(\mu \mathrm{CP})$ techniques. Recently, $\mu \mathrm{CP}$ techniques have been used for micropatterning of various inks, which commonly use poly(dimethylsiloxane) (PDMS) as a "stamp". ${ }^{55}$ However, previous studies reported that PDMS cannot fulfil the requirement of printing polar inks and proteins due to its hydrophobicity. ${ }^{56-59}$ PDMS stamps could be easily hydrophilized by oxygen plasma, however, the effect of the hydrophilization is only temporary, which means the stamps should be hydrophilized every-time prior to the usage. Moreover, PDMS has a number of drawbacks due to its softness such as limitation of pattern shapes and resolution. ${ }^{60}$ Therefore, our $\mathrm{SiO}_{x}$-patterned COP can be a potential candidate for the future $\mu \mathrm{CP}$ "stamp" since it can be easily patterned, has tuneable height difference, and has longer-life hydrophilicity contrast.

Our VUV/(O) affinity-patterning technique is a powerful method, which can provide the templates with an affinity contrast for the further patterning. Without the requirement of photo-active chemicals for oxidation, our method is simple and environmental-friendly. This mild and highly precise treatment showed its application to patterning of silanes and $\mathrm{SiO}_{x}$ on the COP substrates, and for sure it can be extended to various polymer substrates. A wide range of silanes and semiconductors can be patterned on the $\mathrm{VUV} /(\mathrm{O})$-modified polymer substrates through the design of site-selective deposition. We believe that our method is of great interests for future microfabrication. 
(a)

\section{Conclusions}

We demonstrated a confined micropatterning of $\mathrm{SiO}_{x}$ on COP substrate via two steps VUV/(O)-treatment. First, VUV/(O)treatment was experimentally proved to introduce hydrophilic functional groups onto the COP surface. An affinity-patterned COP substrate was prepared by $\mathrm{VUV} /(\mathrm{O})$-treatment through a photomask. TMCTS molecules were chemically adsorbed on the COP substrate by a condensation reaction between the silanol groups and photo-chemically modified COP surface. The resulting siloxane linkages were found to be strong against ultra-sonication. The secondary $\mathrm{VUV} /(\mathrm{O})$-treatment converted TMCTS into $\mathrm{SiO}_{x}$. On the affinity-patterned COP, TMCTS molecules preferentially deposited at the $\mathrm{VUV} /(\mathrm{O})$-modified regions, forming TMCTS micron/submicron-sized patterns with a good fidelity to the photomask. The secondary VUV/(O)treatment converted the TMCTS patterns into $\mathrm{SiO}_{x}$ patterns, and the bare part of COP surface was etched to a depth of $\sim 150 \mathrm{~nm}$. High-quality $\mathrm{SiO}_{x}$ patterns formed on polymer substrates are of great interests for flexible microdevices. Since (b)

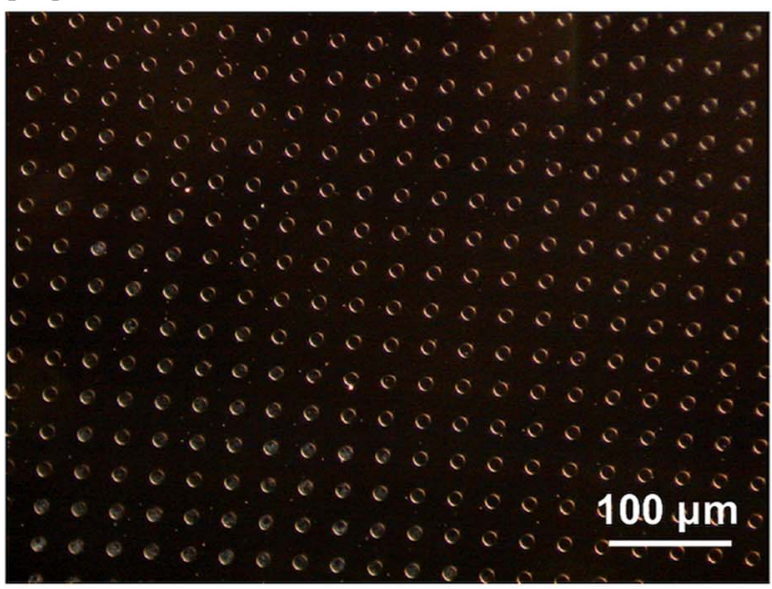

(d)

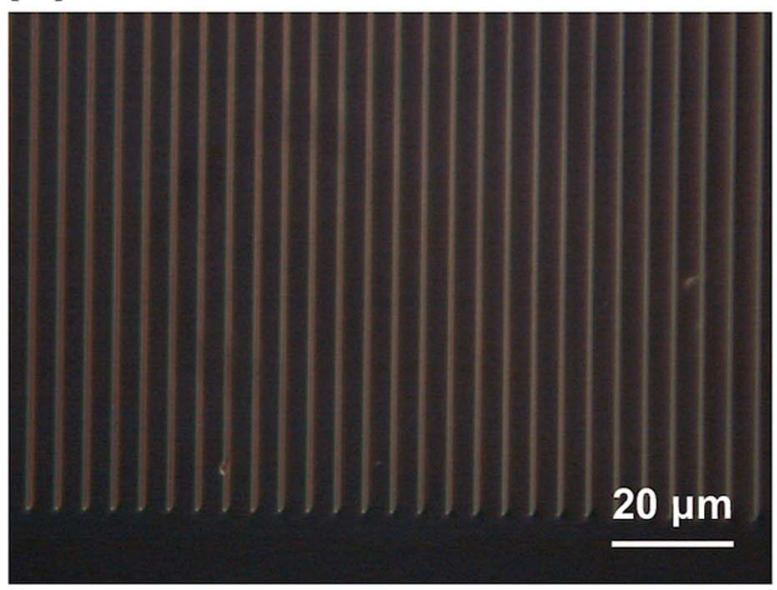

Fig. 9 Optical micrographs of resulting silica micro arrays. The circular patterns with a diameter of $10 \mu \mathrm{m}$ are shown by (a) bright field image and (b) dark field image. The line patterns with (interval, line width) of ( $4 \mu \mathrm{m}, 0.5 \mu \mathrm{m})$ are shown by (c) bright field image and (d) dark field image.

\section{Conflicts of interest}

There are no conflicts to declare.

\section{Acknowledgements}

This work was partially supported by JSPS KAKENHI grant number JP15H02297. Cheng-Tse Wu acknowledges the Japan-

Taiwan Exchange Association for financial support.

the resolution is achievable in sub- $\mu \mathrm{m}$ scale, our $\mathrm{SiO}_{x}$ patterns 作 in $\mu$ CP techniques. Our VUV/(O)-patterning technique and the pos peres aterning techniques and microdevice fabrication for optics, microelectronics, sensors and biotechnology. 


\section{Notes and references}

1 Y. Liu, K. He, G. Chen, W. R. Leow and X. Chen, Chem. Rev., 2017, 117, 12893-12941.

2 S. Park, G. Wang, B. Cho, Y. Kim, S. Song, Y. Ji, M. Yoon and T. Lee, Nat. Nanotechnol., 2012, 7, 438-442.

3 J. S. Discrete, K. Nomura, H. Ohta, A. Takagi and T. Kamiya, Nature, 2004, 432, 3383-3386.

4 Y. H. Kim, J. S. Heo, T. H. Kim, S. Park, M. H. Yoon, J. Kim, M. S. Oh, G. R. Yi, Y. Y. Noh and S. K. Park, Nature, 2012, 489, 128-132.

5 B. K. Song, J. Noh, T. Jun, Y. Jung, H. Kang and J. Moon, Adv. Mater., 2010, 22, 4308-4312.

6 J. Wang, W. Weng, M. Tsai, M. Lee, S. Horng, T. Perng, C. Kei, C. Yu and H. Meng, J. Mater. Chem., 2010, 20, 862-866.

7 P. C. With, U. Helmstedt, S. Naumov, A. Sobottka, A. Prager, U. Decker, R. Heller, B. Abel and L. Prager, Chem. Mater., 2016, 28, 7715-7724.

8 S. Bai, W. Wu, Y. Qin, N. Cui, D. J. Bayerl and X. Wang, Adv. Funct. Mater., 2011, 21, 4464-4469.

9 S. Kim, H. Y. Jeong, S. K. Kim, S. Choi and K. J. Lee, Nano Lett., 2011, 11, 5438-5442.

10 Y. S. Rim, H. Chen, Y. Liu, S. H. Bae, H. J. Kim and Y. Yang, ACS Nano, 2014, 8, 9680-9686.

11 A. Narazaki, Y. Kawaguchi, H. Niino, M. Shojiya, H. Koyo and K. Tsunetomo, Chem. Mater., 2005, 17, 6651-6655.

12 U. Schulz and N. Kaiser, Prog. Surf. Sci., 2006, 81, 387-401.

13 S. Gan, P. Yang and W. Yang, Sci. China: Chem., 2010, 53, 173-182.

14 R. H. Jin and J. J. Yuan, Adv. Mater., 2009, 21, 3750-3753.

15 X. Ding, Y. Kawaguchi, T. Sato, A. Narazaki, R. Kurosaki and H. Niino, J. Photochem. Photobiol., A, 2004, 166, 129-133.

16 H. Ikeda, S. Fujino and T. Kajiwara, J. Am. Ceram. Soc., 2011, 94, 2319-2322.

17 K. Q. Le, M. Nixon and J. Bai, IEEE Journal of Photovoltaics, 2015, 5, 1125-1129.

18 S. Y. Chou, C. Keimel and J. Gu, Nature, 2002, 417, 835-837.

19 B. D. Choudhury, R. Casquel, M. J. Bañuls, F. J. Sanza, M. F. Laguna, R. Puchades, A. Maquieira, C. A. Barrios and S. Anand, Opt. Mater. Express, 2014, 4, 3894-3899.

20 J. K. Lee and S. M. Kang, Bull. Korean Chem. Soc., 2013, 34, 353-354.

21 I. Saaem, K. Ma, A. N. Marchi, T. H. Labean and J. Tian, ACS Appl. Mater. Interfaces, 2010, 2, 491-497.

22 A. Hozumi, H. Sugimura, K. Hiraku, T. Kameyama and O. Takai, Nano Lett., 2001, 1, 395-399.

23 D. J. Kim, K. B. Lee, T. G. Lee, H. K. Shon, W. J. Kim, H. J. Paik and I. S. Choi, Small, 2005, 1, 992-996.

24 K. Koumoto, S. Seo, T. Sugiyama, W. S. Seo and W. J. Dressick, Chem. Mater., 1999, 11, 2305-2309.

25 Y. J. Kim, Y. Taniguchi, K. Murase, Y. Taguchi and H. Sugimura, Appl. Surf. Sci., 2009, 255, 3648-3654.

26 H. Shinohara, J. Mizuno and S. Shoji, Sens. Actuators, A, 2011, 165, 124-131.

27 P. Yang, J. Yuan and W. Tai, Polymer, 2003, 44, 7157-7164.
28 P. Yang, M. Yang, S. Zou, J. Xie and W. Yang, J. Am. Chem. Soc., 2007, 129, 1541-1552.

29 P. Yang, S. Zou and W. Yang, Small, 2008, 4, 1527-1536.

30 Y. Tu, T. Utsunomiya, T. Ichii and H. Sugimura, ACS Appl. Mater. Interfaces, 2016, 8, 10627-10635.

31 A. I. A. Soliman, T. Ichii, T. Utsunomiya and H. Sugimura, Soft Matter, 2015, 11, 5678-5687.

32 H. Sugimura, K. Lee, H. Sano and R. Toyokawa, Colloids Surf., A, 2006, 285, 561-566.

33 H. Sugimura, L. Hong and K.-H. Lee, Jpn. J. Appl. Phys., 2005, 44, 5185-5187.

34 H. Pizem, O. Gershevitz, Y. Goffer, A. A. Frimer, C. N. Sukenik, U. Sampathkumaran, X. Milhet, A. Mcilwain, M. R. De Guire, M. A. B. Meador and J. K. Sutter, Chem. Mater., 2005, 17, 3205-3213.

35 Y. Masuda, T. Sugiyama, W. S. Seo and K. Koumoto, Chem. Mater., 2013, 15, 2469-2476.

36 A. Hozumi, H. Inagai, Y. Yokogawa and T. Kameyama, Thin Solid Films, 2003, 437, 89-94.

37 T. Utsunomiya, T. Kanzawa, T. Ichii and H. Sugimura, Thin Solid Films, 2017, 638, 28-33.

38 R. P. Gandhiraman, C. Volcke, V. Gubala, C. Doyle, L. Basabe-Desmonts, C. Dotzler, M. F. Toney, M. Iacono, R. I. Nooney, S. Daniels, B. James and D. E. Williams, J. Mater. Chem., 2010, 20, 4116-4127.

39 O. P. Khatri, H. Sano, K. Murase and H. Sugimura, Langmuir, 2008, 24, 12077-12084.

40 Y. Kim, J. Han, H. Sano, K. Lee, K. Noda, T. Ichii, K. Murase, K. Matsushige and H. Sugimura, Appl. Surf. Sci., 2009, 256, 1507-1513.

41 N. De Geyter, R. Morent and C. Leys, Surf. Interface Anal., 2008, 40, 608-611.

42 H. Tada, Langmuir, 1995, 11, 3281-3284.

43 A. Grill and D. A. Neumayer, J. Appl. Phys., 2003, 94, 66976707.

44 Y. H. Kim, M. S. Hwang, H. J. Kim, J. Y. Kim and Y. Lee, J. Appl. Phys., 2001, 90, 3367-3370.

45 Y. Lin, T. Y. Tsui and J. J. Vlassak, J. Electrochem. Soc., 2006, 153, F144.

46 M. J. Loboda, C. M. Grove and R. F. Schneider, J. Electrochem. Soc., 1998, 145, 2861-2866.

47 M. Albrecht and C. Blanchette, J. Electrochem. Soc., 1998, 145, 4019-4025.

48 E. McCafferty and J. P. Wightman, Surf. Interface Anal., 1998, 26, 549-564.

49 A. I. A. Soliman, T. Utsunomiya, T. Ichii and H. Sugimura, Langmuir, 2018, 34, 13162-13170.

50 K. T. Queeney, M. K. Weldon, J. P. Chang, Y. J. Chabal, A. B. Gurevich, J. Sapjeta and R. L. Opila, J. Appl. Phys., 2000, 87, 1322-1330.

51 C. Martinet and R. Devine, J. Appl. Phys., 1995, 77, 43434348.

52 R. Tian, O. Seitz, M. Li, W. Hu, Y. J. Chabal and J. Gao, Actual Probl. Econ., 2010, 26, 4563-4566.

53 H. Tada, K. Nakamura and H. Nagayama, J. Phys. Chem., 1994, 98, 12452-12457.

54 D. Neshev and I. Aharonovich, Light: Sci. Appl., 2018, 7, 1-5. 
55 T. Kaufmann and B. J. Ravoo, Polym. Chem., 2010, 1, 371387.

56 C. Donzel, M. Geissler, A. Bernard, H. Wolf, B. Michel, J. Hilborn and E. Delamarche, Adv. Mater., 2001, 13, 11641167.

57 D. C. Trimbach, M. Al-hussein, W. H. De Jeu, M. Decre, D. J. Broer and C. W. M. Bastiaansen, Langmuir, 2004, 20, 4738-4742.
58 H. Li, J. Zhang, X. Zhou, G. Lu, Z. Yin, G. Li, T. Wu, F. Boey, S. S. Venkatraman and H. Zhang, Langmuir, 2010, 26, 56035609.

59 T. Das, S. K. Mallick, D. Paul, S. K. Bhutia and T. K. Bhattacharyya, Colloid Interface Sci., 2007, 314, 71-79. 60 G. Csucs, T. Künzler, K. Feldman, F. Robin and N. D. Spencer, Langmuir, 2003, 19, 6104-6109. 